\title{
Caractérisation structurale et morphologique de couches minces épitaxiées de ZnSe déposées sur GaAs
}

\author{
M. Robino, A. Chergui, J.L. Deiss, J.L. Loison et J.P. Vola \\ Groupe d'Optique Non-Linéaire et d'Optoélectronique, Institut de Physique et Chimie des Matériaux de \\ Strasbourg, UM 380046, CNRS-ULP-EHICS, 23 rue du Loess, 67037 Strasbourg cedex, France
}

\begin{abstract}
Résumé: Des couches minces épitaxiées de $\mathrm{ZnSe}$ ont été déposées par ablation laser sur des substrats monocristallins de GaAs. Linfluence de la température du substrat ainsi que son état de surface sur la qualité structurale de ces couches a tout d'abord été étudiée par Diffraction de Rayons X (XRD). Une épitaxie complète de $\mathrm{ZnSe}$ a été obtenue pour des températures de substrat $>420^{\circ} \mathrm{C}$. La morphologie de la surface libre de ZnSe a également été étudiée par Microscopie à Force Atomique (AFM) et Microscopie Electronique à Balayage (MEB). La qualité structurale et morphologique de ces couches a été comparée à celle de couches minces de ZnSe obtenues par Epitaxie en Phase Vapeur à partir d'Organo-Métalliques (MOCVD) ou par Epitaxie par Jets Moléculaires (MBE). On a pu constater que l'ablation laser (PLA) est une technique de dépôt qui permet d'obtenir des couches épitaxiées de qualité comparable à celle de couches préparées par MOCVD et MBE.
\end{abstract}

\begin{abstract}
Thin epitaxial layers of ZnSe have been deposited on singlecrystalline GaAs substrate by Pulsed Laser Ablation (PLA). The influence of the substrate temperature and its surface state on the structural quality of these layers has been first studied by X-ray diffraction (XRD). A full epitaxial growth of ZnSe has been obtained for substrate temperatures $>420^{\circ} \mathrm{C}$. The surface morphology of $\mathrm{ZnSe}$ has also been studied by Atomic Force Microscopy (AFM) and Scanning Electron Microscopy (SEM). The structural and morphological quality of these ZnSe epilayers on GaAs has been compared to these of epilayers produced by Metal Organic Chemical Vapor Deposition (MOCVD) or by Molecular Beam Epitaxy (MBE). We can notice that the Pulsed Laser Ablation (PLA) is an alternative technique which allows to prepare $\mathrm{ZnSe}$ epilayers of a quality comparable to that of the layers obtained by MOCVD or MBE
\end{abstract}

\section{INTRODUCTION}

Le séléniure de zinc ( $\mathrm{ZnSe}$ ) constitue avec le sulfure de zinc un des matériaux potentiellement intéressant pour l'optoélectronique, du fait de sa largeur de bande interdite importante, $\mathrm{E}_{\mathrm{g}}=$ $2,7 \mathrm{eV}(3,4 \mathrm{eV}$ pour $\mathrm{ZnS})$. Les applications possibles de ces matériaux concernent la réalisation de dispositifs optiques comme les commutateurs optiques bistables, les diodes électroluminescentes ou les diodes laser dans le bleu.

Les méthodes usuelles de dépôt en couches minces sont l'épitaxie en phase vapeur à partir d'organo-métalliques (MOCVD) et l'épitaxie par jets moléculaires (MBE) [1,2]. Cependant, la technique de dépôt par Ablation Laser Pulsé (PLA) apparaît comme une technique alternative et versatile pour obtenir des couches minces à partir de matériaux très divers, des métaux aux céramiques [3]. Cette méthode de dépôt doit son développement et sa notoriété au fait qu'elle est la seule technique permettant d'obtenir des couches minces supraconductrices $\mathrm{HT}_{\mathrm{c}}$ stoechiométriques. Cette technique PLA présente un certain nombre d'avantages qui permettent aussi d'envisager son utilisation pour le dépôt de couches minces semiconductrices, à savoir: $[4,5,6]$ 
- le transport stoechiométrique du matériau de la cible vers le substrat,

- la croissance de couches avec une précision atomique $(\approx 1 \AA /$ impulsion $)$,

- l'obtention de couches minces très lisses,

- la croissance de couches à des températures faibles.

Nous présentons dans ce travail les résultats de nos études sur l'élaboration de couches minces de ZnSe déposées par PLA sur des substrats monocristallins de GaAs (100). La qualité cristalline et morphologique de ces couches minces épitaxiées est analysée par des techniques de diffraction $X$, de microscopie électronique et de microscopie à force atomique (MEB, AFM) et comparée aux couches minces obtenues par MOCVD, élaborées par R.L. Aulombard et son équipe au Groupe d'Etude des Semiconducteurs de Montpellier [7], et à celles faites par MBE (Solid State Institute, Technion Israël).

\section{ETUDE MORPHOLOGIQUE}

La morphologie de la surface des couches épitaxiées de $\mathrm{ZnSe}$ a tout d'abord été analysée par Microscopie Electronique à Balayage (MEB). Cependant, les images obtenues étaient si peu contrastées que l'utilisation d'une technique offrant une meilleure résolution a été envisagée: la Microscopie à Force Atomique (AFM). Les images présentées (Figures 1 et 2) ont été réalisées en mode contact avec le microscope Digital Instruments Nanoscope III.

\subsection{Topographie de surfaces $(5 \mu \times 5 \mu)$}

Les images AFM faites sur des couches élaborées par PLA montrent que leur surface est très lisse. La rugosité moyenne RMS mesurée sur une surface de $5 \mu \times 5 \mu$ n'est que de 4 à $6 \mathrm{~nm}$. Cette faible rugosité est du même ordre de grandeur que celle des couches obtenues par MBE et bien plus faible que celle des couches élaborées par MOCVD. Cependant, les images de la surface révèlent la présence d'un certain nombre de défauts en forme d'îlots $\left(\approx 2.10^{8} / \mathrm{cm}^{2}\right)$. Sur tous les échantillons étudiés, la taille des îlots varie peu. Ils ont un diamètre compris entre 100 à $150 \mathrm{~nm}$ et une hauteur variant de 25 à $50 \mathrm{~nm}$.

\subsection{Résolution atomique}

Le microscope AFM, utilisé avec un scanner spécial de très grande précision, permet d'étudier de petites surfaces à l'échelle atomique, cependant, ces surfaces doivent être très planes et sans défaut. Nous avons tenté l'expérience sur l'échantillon AL23 obtenu par PLA. Les images faites d'abord sur de grandes surfaces, nous ont montré qu'entre les îlots se trouvaient des endroits très lisses (RMS $=0,6 \mathrm{~nm}$ pour une surface de $500 \mathrm{~nm} \times 500 \mathrm{~nm}$ ). L'exploration de ces régions nous a permis d'obtenir la résolution atomique sur une surface de $10 \mathrm{~nm} \times 10 \mathrm{~nm}$. Nous avons mis en évidence des rangées d'atomes bien alignés dans la direction (110) (Figure 2), contrôlée par une orientation aux rayons $X$ (méthode de Laue). 
a)

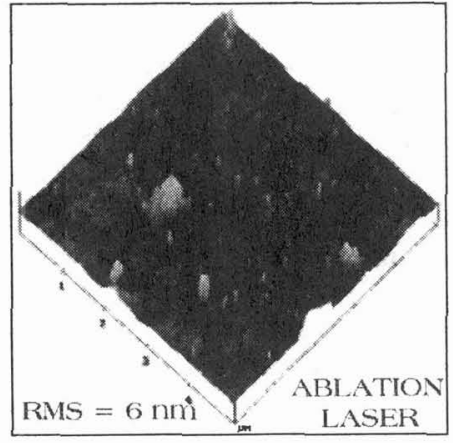

c)

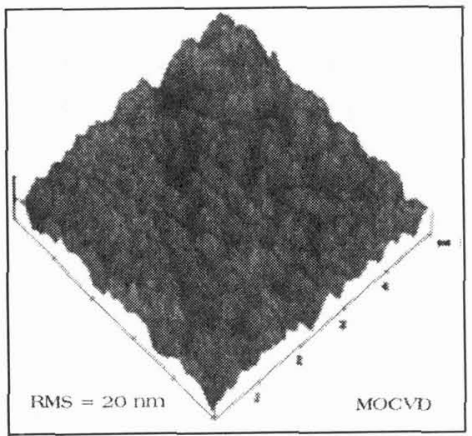

b)

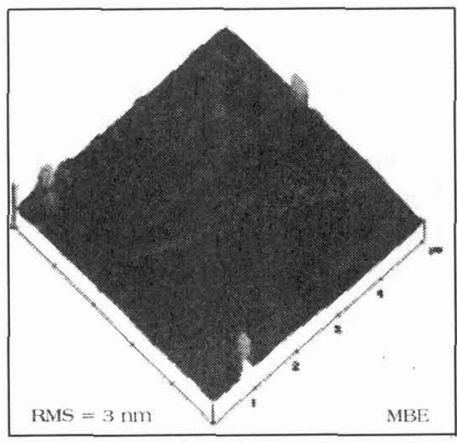

d)

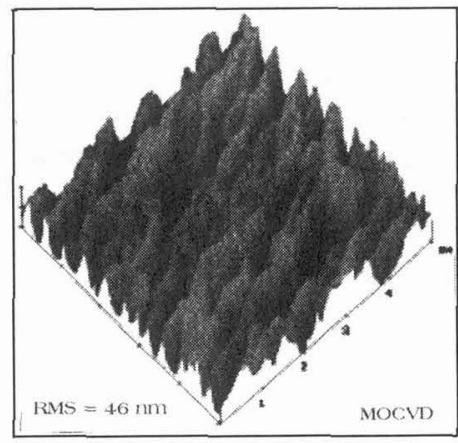

Figure 1: Images, obtenues par Microscopie à Force Atomique, de surfaces de couches minces de ZnSe épitaxiées sur du GaAs (100), élaborées par: a) PLA, b) MBE, c) et d) MOCVD.

AFM images of thin ZnSe layers epitaxied on (100) GaAs substrates, elaborated by: a) PLA, b) MBE, c) and d) MOCVD.

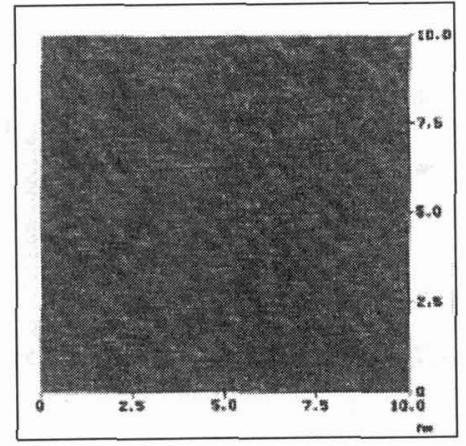

Figure 2: Surface, observée en résolution atomique, d'une couche mince de ZnSe déposée sur un substrat de GaAs (100), élaborée par PLA.

Atomic resolution of a $\mathrm{ZnSe}$ thin layer on (100) GaAs elaborated by PLA. 


\section{ETUDE STRUCTURALE PAR DIFFRACTOMETRIE $X$}

L'étude des couches minces par Diffraction des Rayons X (XRD) s'est révélée être un outil de caractérisation extrêmement fructueux. Cette technique, de mise en oeuvre rapide, conserve les échantillons absolument intacts et permet d'accéder aux caractéristiques importantes suivantes: l'épaisseur, la qualité cristalline et l'état de contrainte des couches.

Les spectres de diffraction de $\mathrm{ZnSe}$ et de GaAs des couches minces de $\mathrm{ZnSe}$ épitaxiées sur GaAs (100) ont été obtenus par diffractométrie $X$, avec une platine goniométrique du type $F$ de Siemens $\left(\theta, 2 \theta\right.$ couplés) et avec une radiation $\mathrm{CuK} \alpha_{1}$ sélectionnée par un monochromateur avant à quartz.

Nous avons procédé à une étude systématique de toutes les couches de ZnSe élaborées par Ablation Laser, permettant d'optimiser les conditions de préparation par attaque chimique de leurs substrats, la température de ceux-ci pendant le dépôt ainsi que les conditions de fréquence et d'énergie des tirs lasers.

Une épitaxie totale n'a été obtenue que lorsque le substrat a été préalablement décapé et chauffé à une température supérieure à $420^{\circ} \mathrm{C}$ durant l'élaboration du film. Dans ces conditions d'élaboration, les pics de diffraction (n00) des couches sont seuls visibles et sont d'une intensité telle que seule une couche monocristalline peut la donner. A une température plus basse et/ou avec un substrat non décapé, l'observation d'un pic unique de diffraction (111) très large et d'intensité très faible semble confirmer la présence d'une couche proche de l'amorphe (Figure 3).

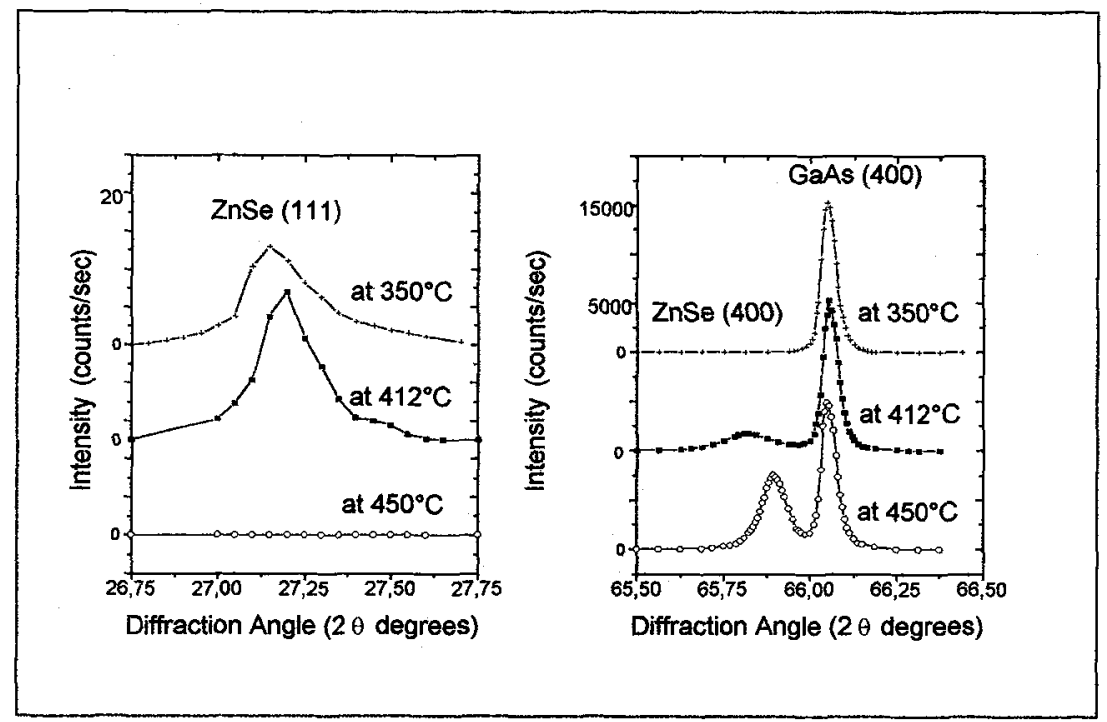

Figure 3: Influence de la température du substrat sur la qualité des couhes de $\mathrm{ZnSe}$ Influence of the substrate temperature on the quality of $\mathrm{ZnSe}$ layers

\subsection{Evaluation de l'épaisseur des couches épitaxiées de ZnSe}

Nous avons évalué l'épaisseur des couches épitaxiées de ZnSe déposées sur des substrats de GaAs (100) à partir du rapport $x$ des intensités des raies de diffraction des rayons $\mathrm{X}$ sur les plans (400) de la couche de ZnSe et du substrat (Figure 3). 
Un faisceau de rayons $\mathrm{X}$, de longueur d'onde $\lambda=1,5405981 \AA\left(\mathrm{CuK} \alpha_{1}\right)$, d'intensité $I_{0}$ est diffracté par le plan (400) de la couche de ZnSe. L'intensité $I^{\text {ZnSe }}$ du faisceau diffracté est égal à:

$$
I^{Z n S e}=P_{(\varepsilon)}^{Z n S e} I_{0}
$$

où $P_{(\varepsilon)}^{2 n S e}$ est le pouvoir réflecteur de la couche de ZnSe d'épaisseur $\varepsilon$.

Comme les couches de $\mathrm{ZnSe}$ que nous caractérisons sont des couches minces dont l'épaisseur est généralement inférieure à $3 \mu$, le faisceau de rayons X est également diffracté par le plan (400) du substrat GaAs. Cependant, l'intensité incidente est alors atténuée à travers la couche de $\mathrm{ZnSe}$. Il en est de même du faisceau diffracté. L'intensité $I^{\text {GaAs }}$ du faisceau diffracté par le substrat GaAs est alors égale à:

$$
I^{G a d s}=P_{\infty}^{G a A s} e^{-\varepsilon / \varepsilon_{a b s}} I_{0}
$$

où $P_{\infty}^{\text {GaAs }}$ est le pouvoir réflecteur du substrat GaAs que l'on peut considérer comme d'épaisseur infinie;

$\mathcal{E}_{a b s}$, appelée distance d'absorption, rend compte de l'atténuation des faisceaux de RX incident et diffracté à travers la couche de $\mathrm{ZnSe}$ [8] et s'écrit:

$$
\varepsilon_{a b s}=\left[\mu\left(\gamma_{i}^{-1}+\gamma_{e}^{-1}\right)\right]^{-1}=6,96 \mu m
$$

avec $\mu=$ coefficient d'absorption linéaire de $\mathrm{ZnSe}$ à la longueur d'onde $\lambda$, $\gamma_{i}$ et $\gamma_{e}$ les sinus des angles d'incidence et d'émergence des $\mathrm{RX}$

Le rapport des intensités des raies de diffraction est donnée en fonction de l'épaisseur $\varepsilon$ de la couche de ZnSe par la relation suivante:

$$
x=\frac{I^{Z n S e}}{I^{\text {GaAs }}}=\frac{P_{(\varepsilon)}^{Z n S e}}{P_{\infty}^{G a A s} e^{-E / \delta_{a b s}}}
$$

L'expression de $P$, établie par W.J. Bartels [8] nous a permis de calculer le pouvoir réflecteur $P_{(\varepsilon)}^{Z n S e}$ du plan (400) de $\mathrm{ZnSe}$ en fonction de l'épaisseur $\varepsilon$ de la couche épitaxiée (Figure 4).

$$
P=\left\{|g|+\left(1+g^{2}\right)^{1 / 2} \operatorname{coth}\left[\frac{\varepsilon}{\varepsilon_{e x t}}\left(1+g^{2}\right)^{1 / 2}\right]\right\}^{-2}
$$

où $g=-\varepsilon_{e x t} / 4 \varepsilon_{\text {abs }}=0,0539$

$\varepsilon_{\text {ext }}$, appelée distance d'extinction est égale à: $\varepsilon_{\text {ext }}=\frac{V\left(\gamma_{i} \gamma_{e}\right)^{1 / 2}}{r_{e} \lambda|F|}=1,5 \mu m$

$V=$ volume de la maille cubique de $\mathrm{ZnSe}$ (paramètre cristallin $\mathrm{a}=5,6676 \AA$ )

$r_{e}=e^{2} / m c^{2}=2.818 \times 10^{-5} \AA$

$F=$ facteur de structure du plan (400) de $\mathrm{ZnSe}=155,10$

Les pouvoirs réflecteurs $P_{(\varepsilon)}^{Z i S e}$ et $P_{\infty}^{2 n S e}$ sont donc égaux à: 


$$
\begin{aligned}
P_{(\varepsilon)}^{Z n S e} & =\left\{0,0539+1,00145 \operatorname{coth}(0,6676 \varepsilon\}^{-2}\right. \\
\text { et } \quad P_{\infty}^{Z n S e} & =0,90 \quad \text { (7) }
\end{aligned}
$$

Par ailleurs, nous avons mesuré le rapport du pouvoir réflecteur $P_{\infty}^{\text {GaAs }}$ de GaAs et de celui $P_{\infty}^{Z n S e}$ de $\mathrm{ZnSe}$ en mesurant le rapport des intensités de diffraction obtenues sur chaque face d'un échantillon épitaxié en couche épaisse $(10 \mu)$. Nous avons obtenu:

$$
P_{\infty}^{\text {GaAs }} / P_{\infty}^{\text {Znse }}=1,3
$$

L'utilisation de cette valeur du rapport, obtenue expérimentalement, rend compte du fait que nous sommes en présence de couches non parfaites, et nous permet d'utiliser les équations établies par Bartels bien qu'elles soient calculées pour des couches parfaites. En remplaçant dans l'expression (4) $P_{\infty}^{\text {Gads }}$ par sa valeur déduite de la mesure (8), ainsi que $P_{\infty}^{Z n S e}$ et $P_{(\varepsilon)}^{Z n S e}$ par leurs valeurs respectives calculée en (6) et (7), on obtient le rapport $x$ des intensités de diffraction sous la forme suivante:

$$
x \times 1,17 e^{-0,1437 \varepsilon}=[0,0539+1,00145 \operatorname{coth}(0,6676 \varepsilon)]^{-2}
$$

On peut obtenir l'épaisseur $\varepsilon$ de la couche épitaxiée à partir de cette expression, soit par résolution graphique, soit par des approximations successives. Dans ce dernier cas, on commence par poser $\varepsilon=0$ dans la partie gauche de l'équation (9), d'où l'on déduit une première valeur approchée $\varepsilon_{1}$ de l'épaisseur. Cette valeur est alors réintroduite dans la partie gauche de l'expression (9). Ces itérations convergent assez rapidement, surtout pour des couches très fines, vers la valeur $\varepsilon$ de l'épaisseur.

Cette méthode, utilisant les intensités des spectres de diffraction des RX, s'est révélée très pratique pour l'évaluation systématique de l'épaisseur des couches de ZnSe épitaxiées sur GaAs (100) par PLA ou par MOCVD. La validité de la méthode a été confirmée par des mesures d'épaisseur de quelques couches à l'aide d'un profilomètre, qui ont donné des écarts inférieurs à $10 \%$.

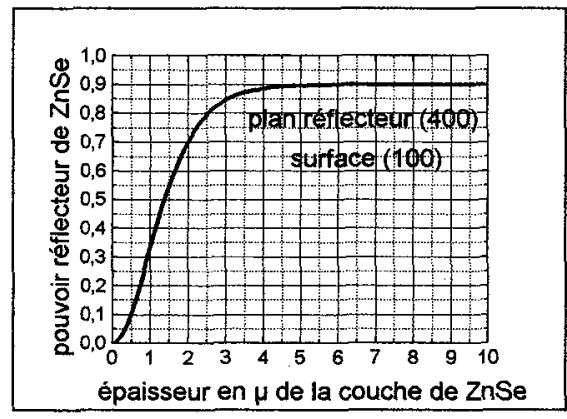

Figure 4: Pouvoir réflecteur $P_{(\varepsilon)}^{Z n S e}$ du plan (400) de ZnSe en fonction de l'épaisseur de la couche mince épitaxiée (100)) Reflecting power of the (400) plane of $\mathrm{ZnSe}$ as a function of the thickness of the epitaxial layer (100) 


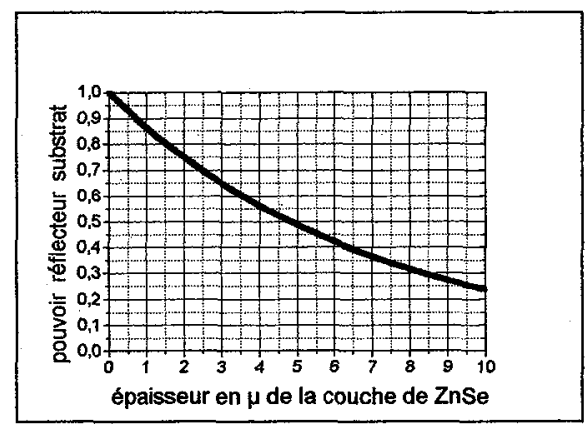

Figure 5: Facteur d'atténuation du pouvoir réflecteur du plan (400) du substrat GaAs en fonction de l'épaisseur de la couche épitaxiée de $\mathrm{ZnSe}$

Attenuation factor of the reflecting power of the (400) plane of $\mathrm{GaAs}$ substrate as a function of the $\mathrm{ZnSe}$ layer thickness.

\subsection{Qualité cristalline des couches épitaxiées de ZnSe}

La valeur de la largeur à mi-hauteur du pic de diffraction (400) de ZnSe est une fonction linéaire de l'inverse de l'épaisseur des couches épitaxiées, mais est également liée à la qualité cristalline des couches. Nous avons porté (Figure 6) les valeurs des mesures de la largeur des pics de diffraction obtenus sur toutes les couches élaborées par PLA en fonction de l'inverse de leur épaisseur, ainsi que des mesures faites, pour comparaison, sur des couches MOCVD. Nous pouvons voir que nos points de mesure sont assez dispersés, ce qui peut être expliqué par les différentes conditions de croissance utilisées (températures, décapages des substrats, cadences et énergies des tirs lasers). mais aussi, on note que ces points se trouvent, pour la plupart, sur une droite de pente légèrement supérieure à celle des points des mesures MOCVD. Les pics de diffraction de ces couches sont donc légèrement plus larges que ceux des couches MOCVD. Cependant, quelques points, correspondant à des couches élaborées récemment par PLA, en modifiant les conditions de dégazage du substrat, montrent que l'on peut atteindre le même niveau de qualité cristalline.

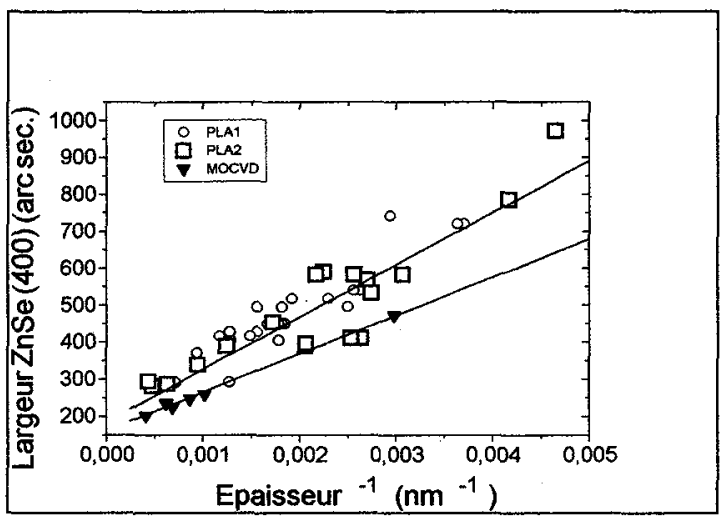

Figure 6: Largeurs à mi-hauteur du pic de diffraction (400) des couches de $\mathrm{ZnSe}$ en fonction de l'inverse de leur leur épaisseur.

Fullwidth at half maximum (FWHM) of the (400) X-ray line of ZnSe epilayers as a function of the inverse of the layer thickness. 


\subsection{Etat de contrainte des couches épitaxiées de ZnSe}

Une étude récente de couches élaborées par MBE [9] attribue la variation, en fonction de l'épaisseur, de l'écart angulaire $\Delta(29)$ entre le pic de diffraction (400) de GaAs et celui de ZnSe à l'état de contrainte des couches épitaxiées sur le substrat. Nous avons tracé (Figure 7) les mesures expérimentales publiées, nos mesures sur des couches MOCVD et celles faites sur toutes les couches PLA..

Les mesures faites sur les couches MOCVD sont en parfait accord avec les mesures MBE publiées. Par contre, les points correspondants aux couches PLA, malgré leur dispersion, sont presque tous situés sous la courbe MBE. Ceci laisse supposer une relaxation des contraintes, à des épaisseurs plus faibles, due à une création importante de défauts.

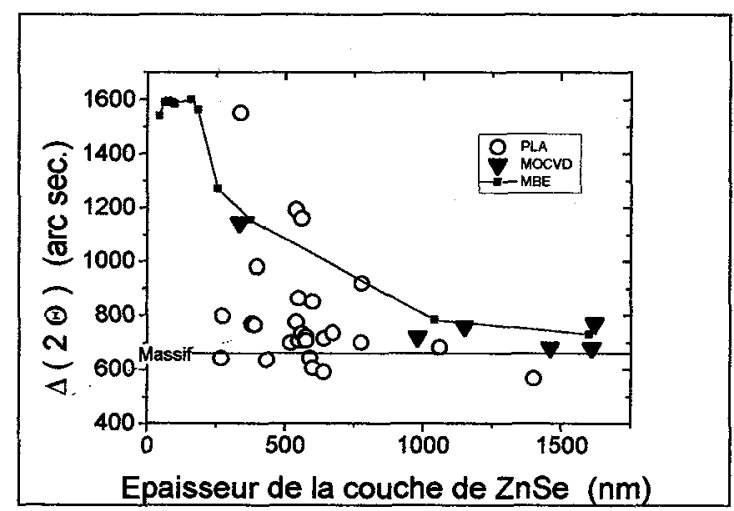

Figure 7: Différence angulaire $\Delta(2 \theta)$ entre les pies de diffraction (400) des couches de $\mathrm{ZnSe}$ et du substrat GaAs en fonction de l'épaisseur de la couche de $\mathrm{ZnSe}$

Angular spacing $\Delta(2 \theta)$ between the (400) X-ray line of $\mathrm{ZnSe}$ layers and of GaAs substrates as a function of the layer thickness.

\section{CONCLUSION}

Les techniques de caractérisation AFM et XRD ont permis d'ajuster les conditions d'élaboration par PLA de couches épitaxiées de ZnSe sur des substrats de GaAs (100) et de suivre l'évolution de leur qualité cristalline.

Les images, obtenues par AFM, des surfaces de ces couches montrent que celles-ci ont une rugosité faible,comparable à celle des couches élaborées par MBE. L'alignement de rangées d'atomes dans la direction (110), observé en résolution atomique, prouve une bonne épitaxie. Les pics de diffraction $\mathrm{X}$ étroits et intenses des plans (n00) et l'absence d'autres réflexions (hkl) indiquent que les couches sont monocristallines et épitaxiées (100) sur le substrat GaAs. Cependant, toutes nos caractérisations montrent que ces couches contiennent quelques défauts de structure dispersés: quelques îlots mis en évidence par AFM et une largeur des pics de diffraction en XRD souvent plus grande que celle observée sur des couches élaborées par MOCVD, ainsi qu'une relaxation plus rapide des contraintes. 
Toutefois, un changement dans le processus d'élaboration (dégazage du substrat plus rapide) a montré une amélioration de la qualité cristalline observée en XRD, ce qui a été confirmé par l'obtention de raies de photoluminescence à basse température.

Finalement, nous avons montré que les conditions initiales de traitement du substrat (procédure de décapage, température de dégazage, durée du dégazage et qualité du vide lors de ce dégazage) sont donc des facteurs essentiels pour l'obtention de couches de bonne qualité optique.[10,11]

\section{Remerciements.}

Les auteurs remercient J.B. Grun pour ses conseils avisés lors de la rédaction de ce travail.

\section{Références}

[1] Wu Yi.H., Toyoda T., Kawakami Y., Fujita Shizuo and Fujita Shigeo, Jpn. J. Appl. Phys. 29 (1990) L144

[2] Gunshor R.L. and Kolodziejski, IEEE J. Quantum Electronics 24 ( 1988) 1744

[3] Shi L., Frankena H.J., Vacuum 40 (1990) 399

[4] McCamy J.W., Lowndes D.H., Budai J.D., Jellison G.E., Herman Jr.I.P. and Kim S., Mat. Res. Symp. Proc. 285 (1993) 471

[5] Rajakarunanayake Y., Luo Y., Adkins B.T. and Compaan A., Mat. Res. Symp. Proc. 285 (1993) 477

[6] McCamy J.W., Lowndes D.H., Budai J.D. Chakoumakos B.C. and Zuhr R.A., Proc. of the Full Meeting of the Materials Research Society, Boston (1991)

[7] Cloitre T., Briot N., Briot O., Gil B.et Aulombard R.L., Mater. Sci. Eng. B21 (1993) 169

[8] Bartels W.J., J. Vac. Sci. B1 (2) (1983) 338

[9] Sou I.K., Mou S.M., Chan Y.W., Xu G.C., Wong G.K.L., J. Cryst. Growth 147 (1995) 39 [10]Deiss J.L., Chergui A., Koutti L., Loison J.L., Robino M. and Grun J.B., Appl. Surf. Sci. 86 (1995) 149

[11]Chergui A., Deiss J.L., Grun J.B., Loison J.L., Robino M. and Beserman R., Third International Conf. on Laser Ablation "Cola 95" Strasbourg (1995) 